\title{
Effect of finite pulse length and laser frequency chirp on HGHG and EEHG seeding
}

\author{
G. Stupakov \\ SLAC National Accelerator Laboratory, Stanford University, Stanford, CA \\ 94309
}

October 25, 2011

\section{INTRODUCTION}

Theoretical studies of high-gain harmonic generation (HGHG) [1] and echoenabled harmonic generation (EEHG) [2, 3] often start from a simplified model in which the beam is assumed infinitely long and longitudinally uniform and the laser induced energy modulation is perfectly sinusoidal and of infinite duration. In such a model the resulting seed has a spectrum consisting of a collection of delta-functions (of zero width) located at the harmonics of the laser frequency. Being a useful tool for study of the seed bunching amplitudes, such a model cannot be used for realistic analysis the spectral properties of the seed.

In this paper we take into account the finite duration of the laser pulse as well as some possible laser phase errors to study their effect on the spectrum of the seed.

\section{DEFINITION OF BUNCHING FACTOR}

Usually the bunching factor $b$ at a given wavenumber $k$ is defined as

$$
b(k)=\frac{1}{N_{0}}\left\langle e^{i k z} N(z)\right\rangle=\frac{1}{N_{0} L} \int_{0}^{L} d z e^{i k z} N(z),
$$


where $N(z)$ is the $1 \mathrm{D}$ density of particles in the beam, $N_{0}$ is the averaged density, $L$ is the length of the beam (so that $N_{0} L$ is the number of particles in the bunch), and angular brackets denote averaging over the bunch length (see, e.g, Eq. (A5) in [3]). This bunching factor is dimensionless and does not depend on the bunch length. This definition is appropriate if one is dealing with a uniform beam, and is not interested in the effects due to either finite length of the bunch of finite duration of the laser pulse. In this paper we will focus on effects due to the finite length of the laser pulse, but will still be assuming a long uniform bunch. In this case it is more convenient to redefine $b(k)$ replacing $N$ by the density perturbation $\delta N$ and dropping the term $1 / L$

$$
b(k)=\frac{1}{N_{0}} \int_{0}^{L} d z e^{i k z} \delta N(z) .
$$

It has now dimension of length, or, if divided by $c$, the dimension of time. One can make it dimensionless by multiplying by the wavenumber $k_{l}$ of the laser. Assuming that $\delta N(z)$ is a localized function, we can extend integration from $-\infty$ to $\infty$, and the integral becomes the Fourier transformation

$$
b(k)=\frac{2 \pi}{N_{0}} \delta \hat{N}(k),
$$

with $\delta \hat{N}(k)=(2 \pi)^{-1} \int_{-\infty}^{\infty} d z e^{i k z} \delta N(z)$.

The so defined bunching function has the following meaning. If one sends a modulated beam with the bunching factor $b(k)$ through a radiator in which a single electron radiates the spectrum $\mathcal{W}(\omega)$ (has dimension of energy per unit frequency), then the spectrum of the beam radiation is $\mathcal{W}(\omega) N_{0}^{2}|b(k)|^{2}$, and the radiated energy is obtained by integration of this quantity over the frequency,

$$
N_{0}^{2} \int d \omega \mathcal{W}(\omega)\left|b\left(\frac{\omega}{c}\right)\right|^{2}
$$


If $\mathcal{W}(\omega)$ is a broad function and $b(\omega / c)$ is a narrow one localized in the vicinity of frequency $\omega_{*}$, then the radiated energy is approximately equal to

$$
N_{0}^{2} \mathcal{W}\left(\omega_{*}\right) \int d \omega\left|b\left(\frac{\omega}{c}\right)\right|^{2},
$$

that is proportional to the integrated over the frequency square of the absolute value of the bunching factor.

\section{VARIATION OF THE LASER AMPLITUDE IN HGHG}

Consider HGHG seeding with a laser pulse which has a Gaussian profile. We assume a uniform beam much longer than the laser pulse. The initial energy distribution of the beam is assumed also Gaussian, given by the distribution

function $f_{0}(p)=N_{0}(2 \pi)^{-1 / 2} e^{-p^{2} / 2}$, where $N_{0}$ is the number of electrons per unit length of the beam, and we use the notation $p=\left(E-E_{0}\right) / \sigma_{E}$ for the dimensionless energy $E$ deviation of a particle, with $E_{0}$ the average energy of the beam and $\sigma_{E}$ the rms energy spread.

Introducing the variable $\zeta=k_{l} z$ where $z$ is the longitudinal coordinate in the beam, one can write the transformation of the initial coordinate $\zeta$ to the final $\zeta^{\prime}$ (resulting from the passage through the energy modulator and the chicane) as

$$
\zeta^{\prime}=\zeta+B p+B A(\zeta) \sin \zeta
$$

where $A(\zeta)=\Delta E / \sigma_{E}$ with $\Delta E$ the local energy modulation in the beam, and $B=R_{56} k_{l} \sigma_{E} / E_{0}$ with $R_{56}$ is the dispersive strength of the chicane. In what follows we assume

$$
A(\zeta)=A_{0} e^{-\zeta^{2} / 2 \sigma_{\zeta}^{2}},
$$

where $\sigma_{\zeta}=\sqrt{2} \sigma_{t} c k_{l}$ with $\sigma_{t}$ the rms length of the laser pulse. Note that $\sigma_{t}$ is the rms length of the Gausian envelope of the laser power while $\sigma_{\zeta}$ is the 
dimensionless rms length of the envelope of the electric field; this explains the factor $\sqrt{2}$ in the equation relating these two quantities. Using (3) we find the bunching factor

$$
b(\kappa)=\frac{1}{k_{l} N_{0}} \int_{-\infty}^{\infty} d p f_{0}(p) \int_{-\infty}^{\infty} d \zeta\left(e^{-i \kappa \zeta^{\prime}(\zeta, p)}-e^{-i \kappa \zeta}\right)
$$

where $\kappa=k k_{l}$. The second term in the last integral is due to the subtraction of the initial uniform density of the beam (so that $b=0$ when $A=B=0$ ); after integration it gives a zero frequency contribution to the bunching factor, $\propto \delta(\kappa)$. Since we are interested in non-zero frequencies, in what follows, we omit this term from the expression for $b(k)$.

Substituting (6) into (8) and carrying out the integration we obtain

$$
\begin{aligned}
k_{l} b(\kappa) & =\frac{1}{N_{0}} \int_{n=-\infty}^{\infty} d p f_{0}(p) \int_{-\infty}^{\infty} d \zeta e^{-i \kappa B p} e^{-i \kappa \zeta-i \kappa B A(\zeta) \sin \zeta} \\
& =e^{-\kappa^{2} B^{2} / 2} \int_{-\infty}^{\infty} d \zeta e^{-i \kappa \zeta-i \kappa B A(\zeta) \sin \zeta} \\
& =e^{-\kappa^{2} B^{2} / 2} \sum_{n=-\infty}^{\infty} \int_{-\infty}^{\infty} d \zeta J_{n}(-\kappa B A(\zeta)) e^{-i(\kappa-n) \zeta} .
\end{aligned}
$$

The bunching spectrum near the $n$-th harmonic, which we denote by $b_{n}$, is given by the $n$-th term in the sum. Using $\Delta \kappa=\kappa-n$, we have

$$
\begin{aligned}
k_{l} b_{n}(\Delta \kappa) & =e^{-\kappa^{2} B^{2} / 2} \int_{-\infty}^{\infty} d \zeta J_{n}(-\kappa B A(\zeta)) e^{-i \Delta \kappa \zeta} \\
& \approx e^{-n^{2} B^{2} / 2} \int_{-\infty}^{\infty} d \zeta J_{n}\left(-n B A_{0} e^{-\zeta^{2} / 2 \sigma_{\zeta}^{2}}\right) e^{-i \Delta \kappa \zeta} \\
& =\sigma_{\zeta} e^{-n^{2} B^{2} / 2} \int_{-\infty}^{\infty} d \xi J_{n}\left(-n B A_{0} e^{-\xi^{2} / 2}\right) e^{-i \Delta \kappa \sigma_{\zeta} \xi},
\end{aligned}
$$

where $\xi=\zeta / \sigma_{\zeta}$.

Our interest now is to evaluate the bunching factor for $n \gg 1$. In an optimized HGHG scheme with the constant amplitude of the laser pulse (that is in the limit $\left.\sigma_{\zeta} \rightarrow \infty\right)$ the maximal value of $b_{n}$ is achieved when $B A_{0} \approx$ 
$1+0.81 n^{-2 / 3}$. With the laser field varying with $\zeta$ as $A_{0} e^{-\zeta^{2} / 2 \sigma_{\zeta}^{2}}$ this may not be an optimal value, so we choose

$$
B A_{0}=r\left(1+0.81 n^{-2 / 3}\right)
$$

where $r$ is a number of order of one. We then have

$$
b_{n}(\Delta \kappa) \approx(-1)^{n} \sigma_{z} e^{-n^{2} r^{2}\left(1+0.81 n^{-2 / 3}\right)^{2} / 2 A_{0}^{2}} F_{n}\left(\Delta \kappa \sigma_{\zeta}, r\right),
$$

with

$$
F_{n}(x, r)=\int_{-\infty}^{\infty} d \xi J_{n}\left(r\left(n+0.81 n^{1 / 3}\right) e^{-\xi^{2} / 2}\right) e^{-i x \xi} .
$$

In the limit $A_{0} \gg 1$ corresponding to the amplitude of modulation much larger than the slice energy spread, one can replace in (12) the exponential factor by 1 ,

$$
\left|b_{n}(\Delta \kappa)\right| \approx \sigma_{z} F_{n}\left(\Delta \kappa \sigma_{\zeta}, r\right)
$$

The integrated over spectrum bunching factor is given by

$$
\int\left|b_{n}(\Delta \kappa)\right|^{2} d \Delta \kappa \approx \sigma_{z}^{2} \int F_{n}\left(\Delta \kappa \sigma_{\zeta}, r\right)^{2} d \Delta \kappa=\frac{\sigma_{z}}{k_{l}} \int F_{n}(x, r)^{2} d x .
$$

The seed spectrum is illustrated by Fig. 1 that shows the normalized function $\left|F_{n}\right|^{2}$ for three values of the harmonic number $n=5,10$ and 20, calculated for $r=1$ (that is for $B A_{0}=1+0.81 n^{-2 / 3}$ ). One can see that higher harmonics have broader spectra. This is due to the fact that higher harmonics are more sensitive to the deviation from the optimization condition $B A=\left(1+0.81 n^{-2 / 3}\right)$. As soon as $A(\zeta)$ deviates from its maximal value $A_{0}$, the local bunching goes down, resulting in shortening of the component of the density modulation carrying the frequency $n \omega_{l}$. Analysis shows that while higher harmonics have broader spectra, they still are Fourier limited pulses (of shorter duration).

Fig. 2 shows two profiles of $\left|F_{10}\right|^{2}$ for $r=1$ and $r=1.1$. Note that $r=1.1$ gives a larger maximal bunching factor. 


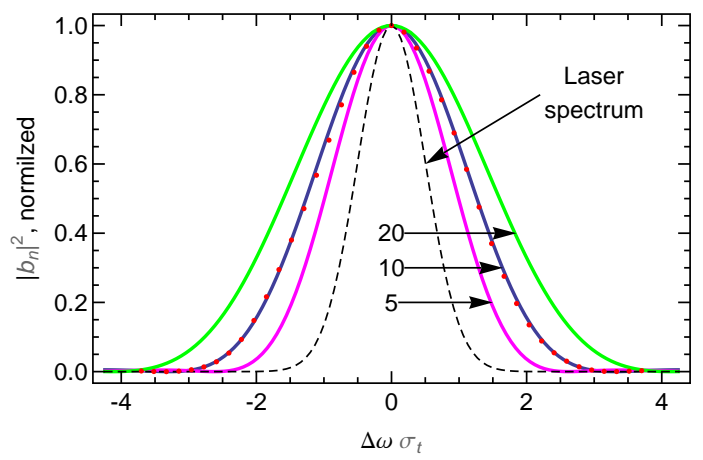

FIG. 1: Bunching spectrum for $n=5,10$ and 20 (larger harmonics have broader profiles) for $r=1$. The dashed curve is the Fourier spectrum of the laser pulse. Each curve is normalized by its maximal value at zero. The frequency deviation $\Delta \omega$ is normalized by the rms laser pulse width (in power) $\sigma_{t}$.

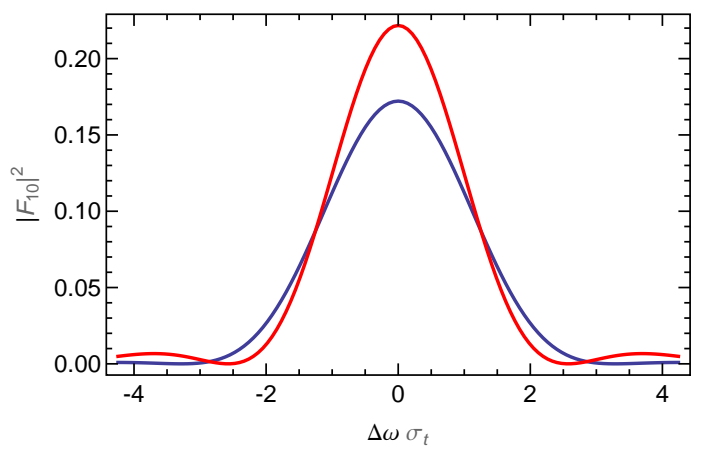

FIG. 2: Plot of functions $\left|F_{10}\right|^{2}$ for $r=1$ (blue) and $r=1.1$ (red).

\section{VARIATION OF THE LASER FREQUENCY}

Let's now take into account possible variation of the laser frequency over the duration of the laser pulse in HGHG seeding. More precisely, we will 
assume a frequency chirp of the laser,

$$
\omega=\omega_{l}+2 \alpha t
$$

which corresponds to the laser phase $\omega_{l} t+\alpha t^{2}$. In laser-speak the chirp is characterized by a spectral phase parabolic profile. Instead of using $\alpha$ (which has dimension of the frequency squared) it is more convenient to introduce the accumulated phase error $\phi$ over the duration of the pulse $\tau$,

$$
\phi=\alpha \tau^{2} .
$$

For the value of $\tau$ we choose the FWHM duration of the laser pulse, $\tau=2.35 \sigma_{t}$, where $\sigma_{t}$ is the rms length of the laser pulse (in power). We will assume that the phase error is small, $\phi \ll 1$.

When such phase errors taken into account Eq. (6) should be replaced by

$$
\zeta^{\prime}=\zeta+B p+B A(\zeta) \sin \left(\zeta+\beta \zeta^{2}\right)
$$

with $\beta=\alpha / \omega_{l}^{2}$, and instead of (9) we now have

$$
k_{l} b(\kappa)=e^{-k^{2} B^{2} / 2} \sum_{n=-\infty}^{\infty} \int_{-\infty}^{\infty} d \zeta J_{n}(-k B A(\zeta)) e^{-i(\kappa-n) \zeta+i n \beta \zeta^{2}} .
$$

Using the optimization (11) we obtain

$$
k_{l} b_{n}(\Delta \kappa)=(-1)^{n} \sigma_{\zeta} e^{-n^{2} r^{2}\left(1+0.81 n^{-2 / 3}\right)^{2} / 2 A_{0}^{2}} G_{n}\left(\Delta \kappa \sigma_{\zeta}, n \beta \sigma_{\zeta}^{2}, r\right),
$$

with

$$
G_{n}(x, y, r)=\int_{-\infty}^{\infty} d \xi J_{n}\left(r\left(n+0.81 n^{1 / 3}\right) e^{-\xi^{2} / 2}\right) e^{-i x \xi+i y \xi^{2}} .
$$

We see that the second argument of the function $G_{n}$ responsible for the phase error is

$$
y=n \beta \sigma_{\zeta}^{2}=\left(\frac{\sqrt{2}}{2.35}\right)^{2} n \phi=0.36 n \phi .
$$


We expect that the phase error effects are negligible when $\phi \ll 1$, and they become essential in the opposite limit.

Fig. 3 shows the normalized bunching factor as a function of the detuning frequency $\Delta \omega$ for the 10th harmonic for different values of the laser frequency chirp characterized by the parameter $n \phi$.

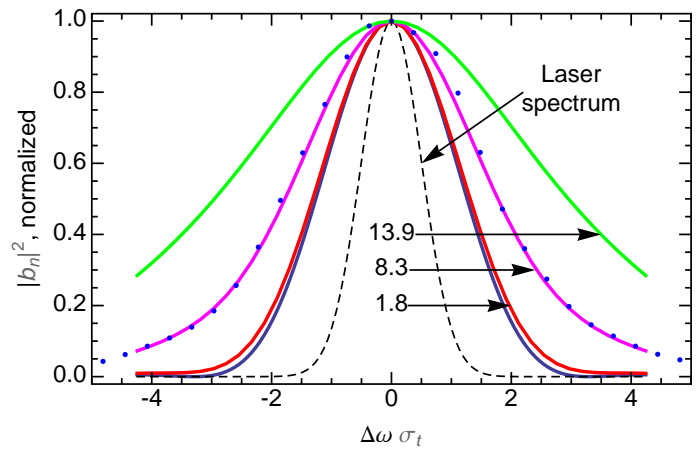

FIG. 3: The bunching factor squared at a function of the detuning for various frequency chirps of the laser. The dash line indicate the spectrum of the laser pulse. The black solid line is the no-chirp case, $\phi=0$. The three broader lines of red, magenta and green have a chirp with the parameter $n \phi$ indicated by arrows.

\section{ANALYSIS}

Let us consider function (21) in the limit of large values of $n$ and use the following representation for the Bessel function of order $\nu \gg 1$,

$$
J_{\nu}\left(\nu+z \nu^{1 / 3}\right)=2^{1 / 3} \nu^{-1 / 3} \operatorname{Ai}\left(-2^{1 / 3} z\right),
$$


where $\mathrm{Ai}$ is the Airy function. In what follows we will assume $r=1$,

$$
\begin{aligned}
& G_{n}(x, y, 1)=\int_{-\infty}^{\infty} J_{n}\left(\left(n+0.81 n^{1 / 3}\right) e^{-\xi^{2} / 2}\right) e^{-i x \xi+i y \xi^{2}} d \xi \\
& =2^{1 / 3} n^{-1 / 3} \int_{-\infty}^{\infty} \operatorname{Ai}\left(-2^{1 / 3} n^{-1 / 3}\left[\left(n+0.81 n^{1 / 3}\right) e^{-\xi^{2} / 2}-n\right]\right) e^{-i x \xi+i y \xi^{2}} d \xi
\end{aligned}
$$

For large $n$ we can expand $e^{-\xi^{2} / 2} \approx 1-\xi^{2} / 2$ because only small values of $\xi$ make dominant contribution

$$
G_{n}(x, y, 1) \approx 2^{1 / 3} n^{-1 / 3} \int_{-\infty}^{\infty} \operatorname{Ai}\left(2^{1 / 3}\left[n^{2 / 3} \xi^{2} / 2-0.81\right]\right) e^{-i x \xi+i y \xi^{2}} d \xi .
$$

This shows that the width of the integrand in variable $\xi$ is of the order of $n^{-1 / 3}$, and hence the spectrum will be wider than the laser spectrum by the same factor. This broadening we see in Fig. 1. Noting that $G_{n}$ is obtained as a Fourier integral, we conclude that the integrand of (29) gives the time profile of the envelope of the $n$-th harmonic:

$$
E_{\text {env }}(t) \propto \operatorname{Ai}\left(2^{1 / 3}\left[n^{2 / 3}\left(t / \sigma_{t}\right)^{2}-0.81\right]\right) .
$$

The plot of the function $E_{\text {env }}(t)^{2}$ normalized by its maximal value at the origin for $n=50,100$ is shown in Fig. 4 .

\section{QUADRATIC CHIRP IN THE BEAM ENERGY}

Let us assume now that instead of the frequency chirp in the laser, we have quadratic energy chirp in the beam

$$
f_{0}(p)=\frac{N_{0}}{\sqrt{2 \pi}} e^{-\left(p+a \zeta^{2}\right)^{2} / 2} .
$$

The quantity $a$ is positive when the beam is accelerated on crest. It is easy to see that this energy chirp produces the same effect as the laser frequency chirp. Indeed, we now need to compute (8) with this distribution function (27):

$$
k_{l} b(\kappa)=\frac{1}{\sqrt{2 \pi}} \int_{-\infty}^{\infty} e^{-\left(p+a \zeta^{2}\right)^{2} / 2} d p \int_{-\infty}^{\infty} e^{-i \kappa B p} e^{-i \kappa \zeta-i \kappa B A(\zeta) \sin \zeta} d \zeta
$$




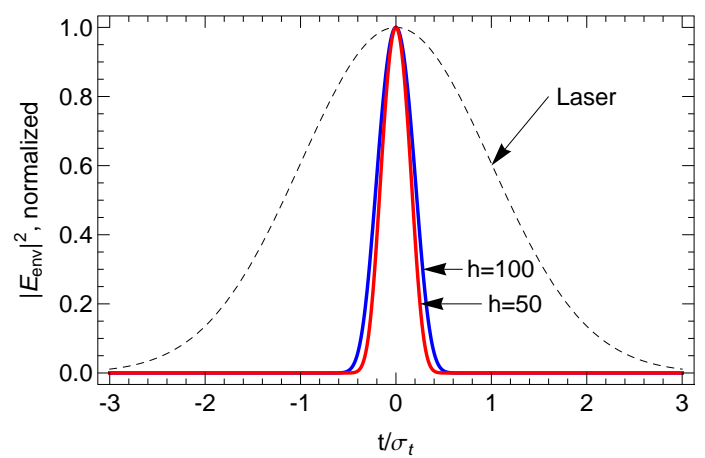

FIG. 4: The envelope profile for 50th (red) and 100th (blue) harmonic. The broken line is the original laser profile. Each curve is normalized by its maximal value at the origin.

Using

$$
\int_{-\infty}^{\infty} e^{-\left(p+a \zeta^{2}\right)^{2} / 2} e^{-i \kappa B p} d p=\sqrt{2 \pi} e^{-\kappa^{2} B^{2} / 2} e^{i \kappa B a \zeta^{2}}
$$

we find

$$
\begin{aligned}
k_{l} b(k) & =e^{-\kappa^{2} B^{2} / 2} \int_{-\infty}^{\infty} d \zeta e^{-i \kappa \zeta+i \kappa B A(\zeta) \sin \zeta} e^{-i \kappa B a \zeta^{2}} \\
& \approx e^{-\kappa^{2} B^{2} / 2} \sum_{n=-\infty}^{\infty} \int_{-\infty}^{\infty} d \zeta J_{n}(-\kappa B A(\zeta)) e^{-i(\kappa-n) \zeta+i n B a \zeta^{2}}
\end{aligned}
$$

This is the same as Eq. (19) with $\beta$ replaced by $B a$.

\section{FINITE LASER PULSE AND FREQUENCY CHIRP IN EEHG}

We now consider the EEHG case assuming a laser pulse given by (7) and a laser frequency chirp (16). In what follows we will take these effects into account only for the second modulator. As for the first one, we will assume that its laser pulse is long enough so that one can neglect variation of the laser 
amplitude over the bunch length. Also, the phase errors of the first laser are likely less important because its role is to generate energy bands in the phase space of the beam which seem less sensitive to the phase errors.

Instead of the HGHG transformation (6) we now have to deal with a more complicated map for the EEHG [3]

$$
\begin{aligned}
\zeta^{\prime} & =\zeta+\left(B_{1}+B_{2}\right) p+A_{1}\left(B_{1}+B_{2}\right) \sin \zeta \\
& +A_{2}(\zeta) B_{2} \sin \left(K \zeta+K B_{1} p+K A_{1} B_{1} \sin \zeta+\beta \zeta^{2}+\psi\right)
\end{aligned}
$$

where $\psi$ is the phase of the second laser, $A_{1}=\Delta E_{1} / \sigma_{E}$ and $A_{2}(\zeta)=\Delta E_{2} / \sigma_{E}$ with $\Delta E_{1}$ and $\Delta E_{2}$ the energy modulation in the first and second undulators, and $B_{1}=R_{56}^{(1)} k_{l} \sigma_{E} / E_{0}, B_{2}=R_{56}^{(2)} k_{l} \sigma_{E} / E_{0}$ with $R_{56}^{(1)}$ and $R_{56}^{(2)}$ the dispersive strengths of the first and second chicanes, respectively. The variable $\zeta$ is now defined as $\zeta=k_{1} z$ with $k_{1}$ the wavenumber of the first laser, and $K=k_{2} / k_{1}$ with $k_{2}$ the wavenumber for the second laser. The parameter $\beta$ is the same as in (18).

We now closely follow the derivation in [3]. Substituting (31) into Eq. (2) gives

$$
\begin{aligned}
k_{l} b(\kappa) & =\frac{1}{N_{0}} \int_{-\infty}^{\infty} d p e^{-i \kappa p\left(B_{1}+B_{2}\right)} f_{0}(p) \int_{-\infty}^{\infty} d \zeta \\
& \times e^{-i \kappa \zeta} e^{-i \kappa A_{1}\left(B_{1}+B_{2}\right) \sin \zeta} e^{-i \kappa A_{2}(\zeta) B_{2} \sin \left(K \zeta+K B_{1} p+K A_{1} B_{1} \sin \zeta+\beta \zeta^{2}+\psi\right)} .
\end{aligned}
$$

Two exponential factors in this equation can be expanded in series:

$$
e^{-i \kappa A_{1}\left(B_{1}+B_{2}\right) \sin \zeta}=\sum_{q=-\infty}^{\infty} e^{i q \zeta} J_{q}\left(-\kappa A_{1}\left(B_{1}+B_{2}\right)\right)
$$

and

$$
\begin{aligned}
& e^{-i \kappa A_{2}(\zeta) B_{2} \sin \left(K \zeta+K B_{1} p+K A_{1} B_{1} \sin \zeta+\beta \zeta^{2}+\psi\right)} \\
& =\sum_{m=-\infty}^{\infty} e^{i m\left(K \zeta+K B_{1} p+K A_{1} B_{1} \sin \zeta+\beta \zeta^{2}+\psi\right)} J_{m}\left(-\kappa A_{2}(\zeta) B_{2}\right)
\end{aligned}
$$


and then, in turn, the factor $e^{i m K A_{1} B_{1} \sin \zeta}$ appearing on the right hand side of Eq. (34) can also be expanded

$$
e^{i A_{1} B_{1} K m \sin \zeta}=\sum_{l=-\infty}^{\infty} e^{i l \zeta} J_{l}\left(A_{1} B_{1} K m\right)
$$

In the limit when $\beta=0$ and $A_{2}$ does not depend on $\zeta$ (that is a very long laser pulse without phase errors) all the terms in (32) that depend on $\zeta$ combine into

$$
e^{i(q+l+m K-\kappa) \zeta}
$$

In this limit the integral does not vanish only if

$$
\kappa=n+m K
$$

where $n=q+l$ is an integer. This means that the echo wavenumber is $k=k_{n, m}=n k_{1}+m k_{2}$. With $\beta \neq 0$ and $A_{2}$ depending on $\zeta, k$ will be close to $k_{n, m}, k=k_{n, m}+\Delta k$, and hence $\kappa=k_{n, m} / k_{1}+\Delta \kappa$, with $\Delta \kappa=\Delta k / k_{1}$. Substituting Eqs. (33)-(35) into Eq. (32) and carrying out integration over $p$ with the help of

$$
\frac{1}{N_{0}} \int_{-\infty}^{\infty} d p e^{-i \kappa p\left(B_{1}+B_{2}\right)+i m K p B_{1}} f_{0}(p)=e^{-\frac{1}{2}\left[\kappa\left(B_{1}+B_{2}\right)-m K B_{1}\right]^{2}},
$$

and also using the identity

$$
J_{s}(a+b)=\sum_{k=-\infty}^{\infty} J_{k}(b) J_{s-k}(a)
$$

we arrive at

$$
\begin{aligned}
b_{n, m}(\Delta \kappa) & =e^{-\frac{1}{2}\left(n B_{1}+(K m+n) B_{2}\right)^{2}} J_{n}\left(-A_{1}\left(n B_{1}+(K m+n) B_{2}\right)\right) \\
& \times \int_{-\infty}^{\infty} d \zeta e^{-i \Delta \kappa \zeta+i m \beta \zeta^{2}+i m \psi} J_{m}\left(-(K m+n) A_{2}(\zeta) B_{2}\right),
\end{aligned}
$$


where $b_{n, m}$ is the bunching factor in the vicinity of $k_{n, m}$.

Let's now assume that $K=1$ (both lasers have the same frequency), $n=-1$ and $m=h+1$, where $h$ is the harmonic number. We denote $b_{-1, h+1}$ by $b_{h}$. Also assume a Gaussian pulse (7) for $A_{2}$ and the optimized value for $A_{0}$ : $(m-1) A_{0} B_{2}=m+0.81 m^{1 / 3}($ see $[3])$ :

$$
\begin{aligned}
b_{h}(\Delta \kappa) & =\sigma_{\zeta} e^{-\frac{1}{2}\left(-B_{1}+h B_{2}\right)^{2}} J_{-1}\left(-A_{1}\left(-B_{1}+h B_{2}\right)\right) e^{i(h+1) \psi} \\
& \times \int_{-\infty}^{\infty} e^{-i \Delta \kappa \sigma_{\zeta} \xi+i(h+1) \beta \sigma_{\zeta}^{2} \xi^{2}} J_{h+1}\left(-\left((h+1)+0.81(h+1)^{1 / 3}\right) e^{-\xi^{2} / 2}\right) d \xi .
\end{aligned}
$$

We see that the dependence of $b_{h}$ versus $\Delta \kappa$ is given by the function $G_{n}\left(\Delta \kappa \sigma_{\zeta},(h+1) \beta \sigma_{\zeta}^{2}, 1\right)$ defined in (21). Hence the analysis developed for the HGHG seeding is also applicable for the EEHG.

\section{ACKNOWLEDGMENTS}

I would like to thank A. Fry for useful discussions.

This work was supported by the U.S. Department of Energy under Contract No. DE- AC02-76SF00515.

[1] L. Yu, Phys. Rev. A 44, 5178 (1991).

[2] G. Stupakov, Phys. Rev. Lett. 102, 074801 (2009).

[3] D. Xiang and G. Stupakov, Phys. Rev. ST Accel. Beams 12, 030702 (2009). 